Ann. Biol. anim., Bioch., Biophys., I965, 5 (4), 45I-468.

\title{
UN NOUVEAU GÈNE, MODIFICATEUR DE LA FORME DES CRETTES EN ROSE, ET SON INGIDENCE POSSIBLE SUR LA FERTILITÉ DES COQS
}

\author{
A. CAVALIE et P. MÉRAT \\ Station de Recherches avicoles, \\ Centre national de Recherches zootechniques, Jouy-en-Josas (Seine-et-Oise).
}

\section{SOMMAIRE}

Un nouveau gène de forme de la crête est mis en évidence sur une population pedigree issue au départ d'un croisement entre Leghorns, Rhode-Island et Wyandottes. Ce gène se manifeste en présence de la crête en rose. Il comporte deux allèles, que nous désignons respectivement par $\mathrm{He}^{+}$(" hérissé ») et $\mathrm{He}^{l}$ ("lisse»). Le premier est dominant. Il produit chez le poussin d'un jour une crête en rose d'aspect granuleux, alors que celle-ci est lisse chez les animaux homozygotes pour le second (cf. fig. I). Chez les adultes, le gène $\mathrm{He}^{+}$s'accompagne d'une crête plus volumineuse, avec des pointes plus nombreuses et plus hautes que son allèle $\mathrm{He}^{l}$. (cf. fig. 2). La différence entre les deux phénotypes est bien tranchée à $\mathrm{r}$ jour, un peu moins bien chez l'adulte.

La pénétrance du récessif à $\mathrm{I}$ jour semble complète chez les o o , mais incomplète chez les $\overrightarrow{0} 0$. Ceci ressort des proportions observées dans les différents types de croisement relatifs à ce facteur (tabl. I).

Chez les 우 우, outre leur accord avec les proportions prévues, les résultats ne montrent pas d'hétérogénéité entre familles (tabl. 2); une telle hétérogénéité se manifeste entre pères pour les proportions chez les $\delta \delta^{*}(\operatorname{tabl} 3)$, suggérant une variabilité génétique de la pénétrance du gène $\mathrm{He}^{l}$ dan ce sexe.

Les données qui précèdent, et des croisements obtenus dans une autre population, avec l'un des parents à crête simple, montrent que le locus en cause est distinct de $\mathrm{R} / r$ (crête en rose, crête simple) et ne présente vraisemblablement pas de linkage avec ce dernier. (tabl. 7).

Des mesures sur le squelette suggèrent que ce gène pourrait influer sur la morphologie du crâne

Une différence appréciable de fertilité se manifeste entre les coqs à crête en rose "hérissée " et ceux à crête "lisse", ces derniers étant supérieurs aux premiers, et plus homogènes (tabl, 9 et Io). simple.

Des recherches sont poursuivies pour savoir si la même différence existe en présence de la crête

Nous n'avons pas trouvé pour l'instant, de différences significatives entre les animaux des deux phénotypes pour les caractères suivants : poids à 8 semaines des coquelets et des poulettes, nombre d'œufs pondus de l'entrée en ponte au $3^{1}$ décembre (pour des poulettes nées au printemps), poids. moyen des œufs, mortalité des jeunes et des adultes (tabl. II). Par contre, la mortalité après le I $8^{\mathrm{e}}$ jour d'incubation est un peu plus grande pour les embryons à crête " hérissée " (tabl. I2). 


\section{INTRODUC'TION}

Le gène $\mathrm{R}$ (crête en rose) est caractérisé par une pénétrance complète (HUTT, I949) et une dominance pratiquement totale (FISHER, I938). Quant à son mode d'expression, on sait que l'apparence extérieure des crêtes en rose présente une certaine diversité (ALDER, I946). Toutefois, on n'a jamais essayé d'analyser sous l'angle génétique les variations correspondantes.

L'objet du présent article est, précisément, la mise en évidence d'un gène modifiant la forme des crêtes rosacées, à partir de l'observation par l'un de nous (A. CAVALIE) de deux types bien tranchés parmi ces crêtes, chez le poussin d'un jour.

L'identification d'un nouveau gène peut permettre, entre autres choses, de compléter ultérieurement la carte chromosomique d'une espèce, spécialement si, comme ici, son expression phénotypique est précoce. Mais son principal intérêt réside sans doute dans une association avec le taux de fertilité des mâles, association que nous discuterons par la suite.

\section{MATÉRIEL ETT MÉTHODES}

Le matériel principal de cette étude était une population pedigree issue, en I96r, de croisements réciproques entre des Leghorns blanches et des coc!s ou poules croisés (Rhode-Island $\times$ Wyandotte). Cette population est désignée sous le nom de «souche $L 22$ ". Les générations ultérieures étaient reproduites en troupeau fermé. Les animaux à crête en rose étaient gardés comme re producteurs, certains d'entre eux étant hétérozygotes $\mathrm{R} r$. Le nombre de parents mâles par génération était de 22 , et celui des femelles d'une centaine ( 5 par coq en général). La reproduction avait lieu en cases pedigree, sans insémination artificielle.

La crête était observée chez le poussin d'un jour, puis revue à l'âge de 8 semaines, et enfin chez les adultes. Le sexe était noté à 8 semaines, et vérifié ${ }_{1} 5$ jours plus tard.

Sur cette population, l'étude porte sur les années ig63, I964 et 1965.

Une deuxième population (souche "Jouy ") fournit quelques données complémentaires, notamment pour le cas où l'un des deux parents est à crête simple (années 1963 et 1964).

\section{RÉSUITATS ET DISCUSSION}

\section{r. Mise en évidence d'un déterminisme monogénique}

a) Phénotypes et proportions observés.

Sur la génération de la souche $L 22$ née au printemps I963, il est apparu que les poussins d'un jour à crête en rose pouvaient être classés en deux catégories. Les uns possédaient une crête d'aspect granuleux, hérissée de petits tubercules, tandis que, chez les autres, la surface était lisse, présentant seulement quelques légères rides longitudinales. Les photographies suivantes (fig. I) montrent l'apparence typique des deux groupes. 

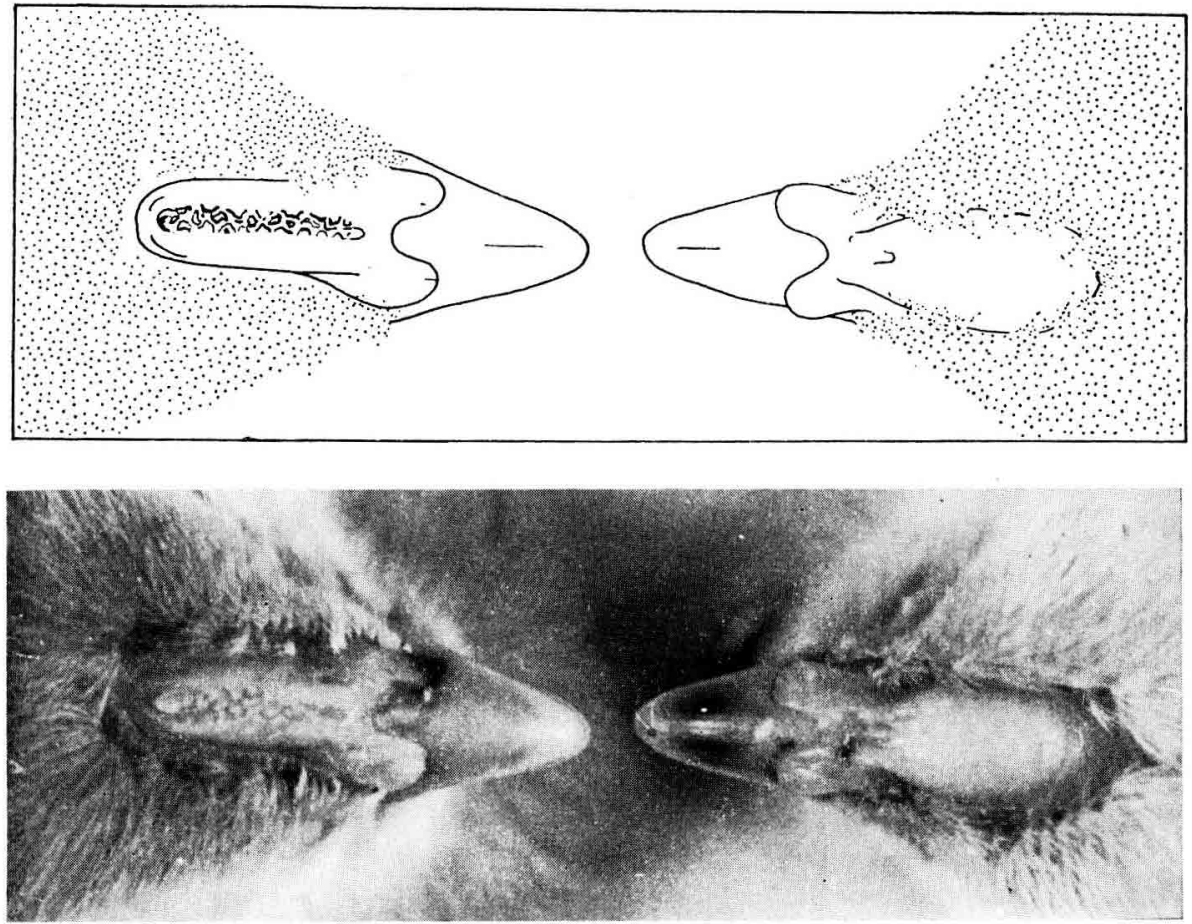

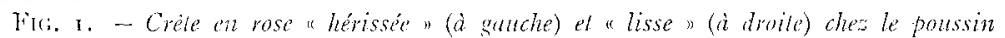
(schém.t et photo)

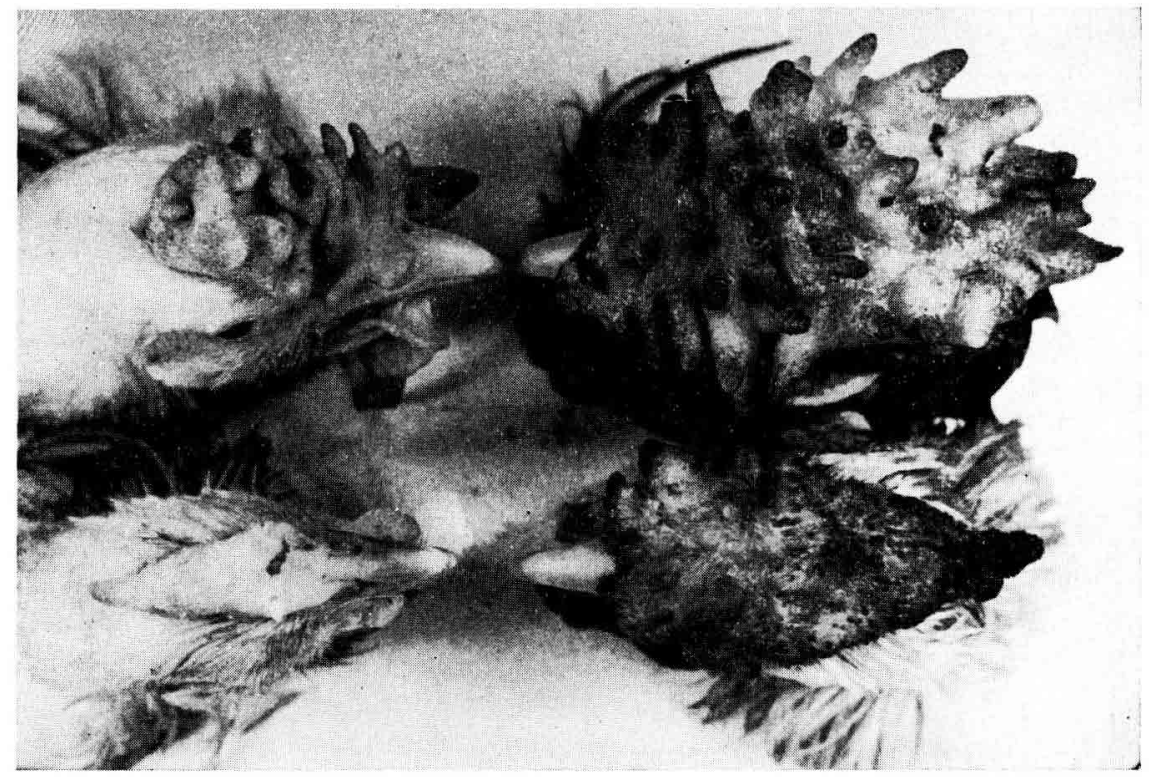

Fig. 2. - Crête "hérissée " (en haut)

et "lisse" (en bas). A droite, cogs; à gauche, poules 
Dans ce qui suit, nous appellerons respectivement " hérissées " et " lisses " ces deux sortes de crêtes.

I a distinction entre les deux, à la naissance, est bien tranchée : le pourcentage de classifications douteuses est faible, n'excédant sans doute pas, par exemple, celui relatif au gène d'emplumement lié au sexe.

Ultérieurement, les crêtes " hérissées " deviennent plus volumineuses que les crêtes "lisses ", avec des pointes plus nombreuses et plus hautes, comme le montrent les photos ci-avant (fig. 2) dans un cas typique. Cependant, la classification, soit à 8 semaines, soit à l'âge adulte, présente davantage d'incertitudes qu'à l'éclosion, surtout chez les coqs. Nous ne tiendrons donc compte que de l'identification à I jour.

En I 964 et I965, en outre, les embryons morts après le I 8 e jour d'incubation ont été observés pour le même caractère. Comme leur classification ne présentait pas, a priori, d'ambiguité, ils ont été inclus dans les proportions présentées plus loin.

La répartition des deux catégories par familles, en 1963 , suggérait 1'hypothèse d'un déterminisme mendélien simple, avec un allèle dominant responsable du caractère " hérissé " et un récessif correspondant au caractère "lisse ".

Les deux années suivantes, le phénotype des parents était connu. Dans l'analyse des proportions observées par type de croisement, nous n'avons retenu, pour vérifier notre hypothèse, que les familles de frères-sœurs d'effectif supérieur à 6 quand l'un des deux parents était " lisse ", et supérieur à 8 quand les deux parents étaient " hérissés ", et, parmi ces familles, nous avons distingué celles qui ne donnaient que des enfants " hérissés » de celles où une ségrégation apparaissait.

Les résultats sont contenus dans le tableau I. Nous y avons omis les poussins de classement douteux. Leur nombre était déjà réduit en Ig64 (I3 97 sur 542 au total, et $29 \sigma^{x} O^{x}$ sur 527), et, en 1965, un classement a été assigné à chaque animal.

Les données du tableau I paraissent bien, à première vue, s'accorder avec l'hypothèse de départ en ce qui concerne les proportions chez les femelles; ils suggèrent, par contre, une pénétrance incomplète du caractère " lisse " chez les mâles. Nous discuterons successivement ces deux points.

\section{b) Interprétation des proportions chez les $\$$ ?.}

Sur Ior poussins femelles issus du croisement $\sigma^{x}$ " lisse " $\times Q$ " lisse ", 2 seulement ont été classés " hérissés ". L'ensemble des données suggère qu'il s'agit, en fait, soit de cas douteux, soit d'erreurs matérielles d'enregistrement.

Cette interprétation est, en effet, renforcée par les proportions voisines de I/I trouvées dans ceux des croisements du typa $\sigma^{x}$ " hérissé " $x q$ " lisse " ou $\sigma^{x}$ "lisse " $\times q$ " hérissée " dans lesquels une ségrégation était observée: les $\chi^{2}$ de conformité vis-à-vis de la proportion théorique, sur le total des deux années, sont respectivement de 0, Igo pour le premier cas $(\mathrm{P}>0,7)$ et de $0,6 \mathrm{I} 7$ pour le second $(\mathrm{P}>0,3)$.

De même, la proportion parmi les croisements du type $\sigma^{*}$ " hérissé " $\times Q$ " hérissé " et qui présentent une ségrégation n'est pas très éloignée de $3 / \mathrm{I}$, malgré un certain excès de $\$ \supsetneq$ "lisses " (29 p. Ioo au lieu de 25 p. Ioo) juste significatif au seuil 5 p. Ioo $\left(\chi^{2}=4,214\right)$. Peut-être cet écart s'explique-t-il par une mortalité embryonnaire un peu plus grande des embryons " hérissés ": Comme il est précisé plus loin, nous avons vérifié ceci, du moins, après le 18 e jour d'incubation. 


\section{TABLEAU I}

Proportions par type de croisement (souche $L$ 22)

\begin{tabular}{|c|c|c|c|c|c|c|c|c|}
\hline \multirow{3}{*}{ Type de croisement } & \multirow{2}{*}{\multicolumn{2}{|c|}{$\begin{array}{l}\text { Nombre de } \\
\text { familles }\end{array}$}} & \multicolumn{6}{|c|}{ Proportion chez les descendants } \\
\hline & & & \multicolumn{2}{|c|}{$a^{x}$} & \multicolumn{2}{|c|}{ 우우 } & \multicolumn{2}{|c|}{ Total } \\
\hline & Pères & Mères & "hérissés" & "lisses" & hérissées" & "lisses" & "hérissés" & "lisses" \\
\hline $\begin{array}{r}o \text { lisse } \times o \text { lisse }: \\
196, \ldots \ldots \ldots \ldots \ldots\end{array}$ & 3 & 5 & 5 & $3^{\prime}$ & 2 & 34 & 7 & $6 \dot{8}$ \\
\hline $1965 \ldots \ldots \ldots \ldots$ & 2 & 9 & 0 & 53 & 0 & 65 & 0 & 118 \\
\hline Total........ & 5 & $1^{\prime} t$ & 5 & 87 & 2 & 99 & 7 & 186 \\
\hline $\begin{array}{l}\sigma \text { hérissé } \times \text { 우 lisse } \\
\text { avec ségrégation : }\end{array}$ & & & & & & & & \\
\hline $1964 \ldots \ldots \ldots \ldots$ & 6 & 18 & 83 & 48 & 78 & $7 t^{\prime}$ & 161 & 122 \\
\hline $1965 \ldots \ldots \ldots \ldots$ & 10 & 20 & 102 & 54 & 94 & 90 & 196 & $1 / 44$ \\
\hline Total ........... & 16 & 38 & 185 & 102 & 172 & $16^{\prime}$ & 357 & 266 \\
\hline sans ségrégation : & & & & & & & & \\
\hline $1964 \ldots \ldots \ldots \ldots$ & 0 & 0 & - & - & - & - & - & - \\
\hline $1965 \ldots \ldots \ldots \ldots$ & 0 & 0 & - & - & - & - & - & - \\
\hline 'Total ............ & 0 & 0 & - & - & - & - & - & - \\
\hline $\begin{array}{c}\text { đlisse } \times \text { q hérissée } \\
\text { avec ségrégation : }\end{array}$ & & & & & & & & \\
\hline $196 \dot{4} \ldots \ldots \ldots \ldots$ & 5 & 16 & $6, t$ & 35 & 72 & 54 & 136 & 89 \\
\hline $1965 \ldots \ldots \ldots \ldots$ & 5 & 17 & $8 ' t$ & 63 & 9 ' & 98 & 178 & 161 \\
\hline Total ........... & 10 & 33 & $1 / 18$ & 98 & 166 & 152 & $31^{\prime} t$ & 250 \\
\hline sans ségrégation : & & & & & & & & \\
\hline $1964 \ldots \ldots \ldots \ldots$ & 1 & 2 & 31 & 0 & 25 & 0 & 56 & 0 \\
\hline $1965 \ldots \ldots \ldots \ldots$ & 0 & 0 & 一 & - & - & - & - & - \\
\hline Total $\ldots . . . \ldots \ldots$ & 1 & 2 & 31 & 0 & 25 & 0 & 56 & 0 \\
\hline $\begin{array}{l}\text { ôhrissé } \times \text { o hérissée } \\
\text { avec ségrégation : }\end{array}$ & & & & & & & & \\
\hline 1964. & 10 & 29 & 202 & 27 & 173 & 50 & 375 & 83 \\
\hline $1965 \ldots$ & 9 & 21 & 97 & 46 & 123 & 67 & 220 & 113 \\
\hline Total ............ & 19 & 50 & 299 & 73 & 296 & 123 & 595 & 196 \\
\hline sans ségrégation : & & & & & & & & \\
\hline $196^{\prime}+\ldots \ldots \ldots \ldots$ & 5 & 9 & 69 & 0 & 74 & 0 & 143 & 0 \\
\hline $1965 \ldots \ldots \ldots \ldots$ & 6 & 10 & 86 & 0 & 83 & 0 & 169 & 0 \\
\hline Total $\ldots \ldots \ldots \ldots$ & 11 & 19 & 155 & 0 & 157 & 0 & 312 & 0 \\
\hline
\end{tabular}



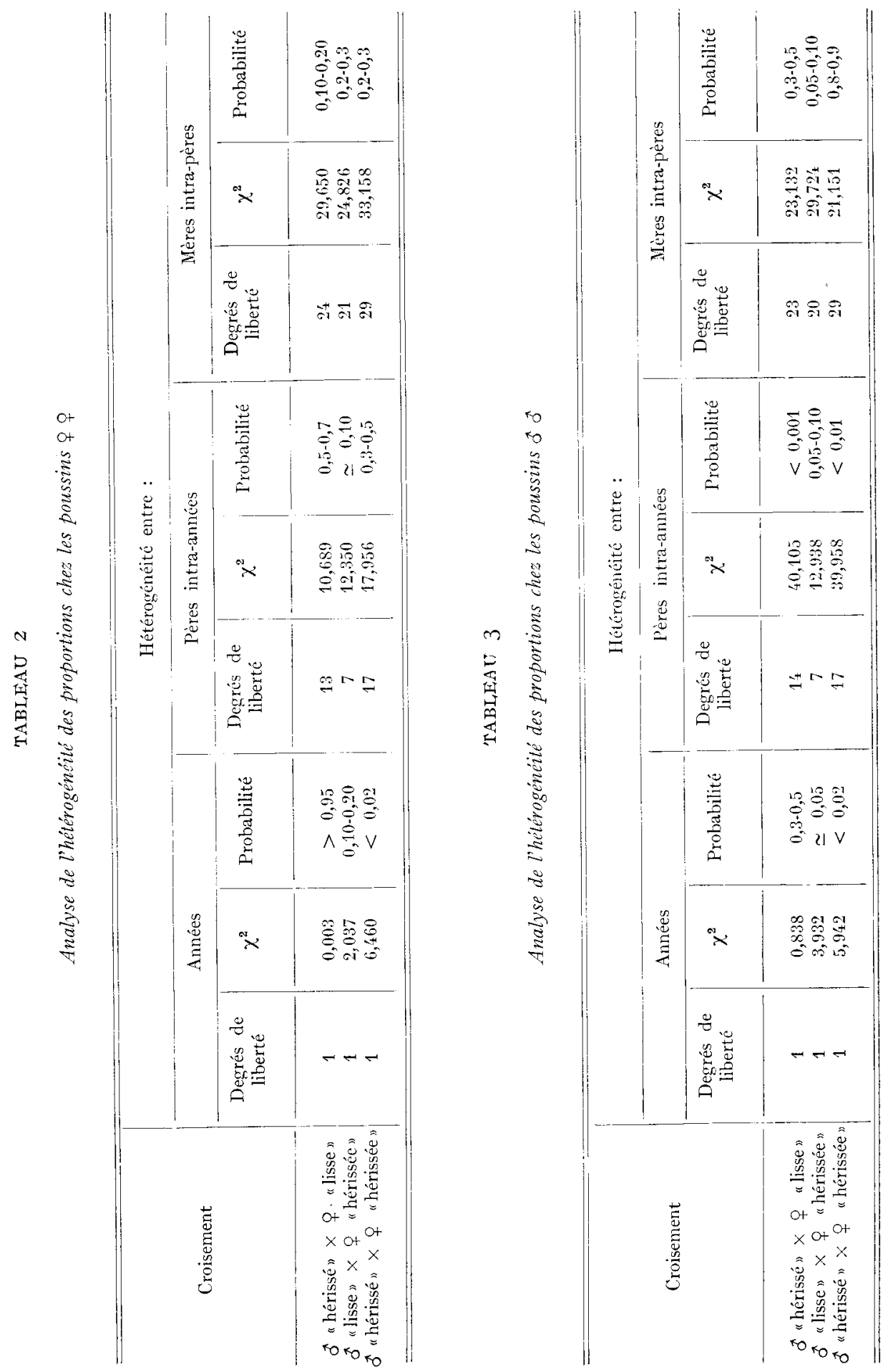
Du fait de la pénétrance vaisemblablement incomplète du phénotype récessif chez les mâles, il fallait vérifier le génotype supposé des pères " hérissés ". D'après les proportions de leur descendance en croisement avec des poules "lisses ", nous n'en avons trouvé qu'un seul qui doit être génétiquement homozygote pour le gène "lisse ": les données correspondant à sa descendance n'ont pas été comptées dans le tableau I.

Qu'il s'agisse des croisement où l'un des parents est "hérissé " et l'autre "lisse ", ou de ceux où les deux sont "hérissés ", nous n'avons trouvé d'hétérogénéité des proportions chez les poussins femelles, ni entre familles de pères, ni entre familles de mères. C'est ce qu'indique le tableau 2, qui contient l'analyse des $\chi^{2}$ d'hétérogénéité suivant ces critères de classification.

La seule variation suggérée, entre années, concerne le dernier type de croisement, où l'année 1965 comporte un excès de femelles "lisses "par rapport à la prévision de $\mathrm{I} / 4$, correspondant peut-être à une différence de mortalité embryonnaire entre zygotes " hérissés et "lisses" (cf. p. 455).

\section{c) Proportions chez les poussins mâles.}

Il y a évidemment, dans l'ensemble, un défaut de crêtes " lisses ", chez les poussins mâles, par rapport aux prévisions. Les $\chi^{2}$ des totaux (par rapport aux proportions prévues $\mathrm{I} / \mathrm{I}$ ou $3 / \mathrm{I}$ ) pour chacun des trois types de croisenent donnant une ségrégation, sont respectivement de 24,003 , de to, I63 et de 5,735 pour I degré de liberté. Les probabilités correspondantes sont inférieures à 0,01 , à 0,001 et à 0,02 .

Ce défaut semble, à première vue, inégal suivant le croisement envisagé : il apparaît moins important dans les accouplements où les deux parents sont " hérissés " que dans les autres. En outre, les croisements $\sigma$ " lisse " $\times \subsetneq$ "lisse " donnent assez peu de descendants mâles " hérissés ». En fait, ces différences de proportion doivent non pas être liéess au type de croisement, mais refléter des variations entre familles. C'est ce que fait apparaître l'analyse du $\chi^{2}$ (tabl. 3 ).

On ne constate pas d'hétérogénéité significative entre mères. D'autre part, vu l'importance de celle qui se manifeste entre pères, l'hétérogénéité entre années doit être testée par le rapport entre le $\chi^{2}$ correspondant et celui entre pères, divisé par son nombre de degrés de liberté. Il devient alors clair qu'il n'y a pas d'effet significatif attribuable à un facteur " années".

L'existence de différences entre familles fait penser à la présence de gènes modificateurs, à effet limité au sexe mâle. Il n'est pas possible, pour l'instant, de dire quels gènes pourraient intervenir. En tous cas, nous n'avons pas trouvé de relation nette entre le rapport de ségrégation chez les mâles et leur génotype homozygote et hétérozygote au locus $\mathrm{R}$, comme l'indique le tableau 4.

Cette hypothèse de gènes modificateurs est la plus vraisemblable ; cependant, nous n'avons pas encore pu la vérifier par des tests de descendance.

Nous pouvons, de toutes façons, écarter la possibilité d'une mortalité plus grande des embryons mâles de type "lisse ": Le taux d'éclosion était assez bon pour les deux années étudiées, et nous avons vérifié qu'il n'était pas inférieur pour les familles à excès significatif de mâles " hérissés " (au seuil 5 p. IO0), comparées aux autres ; il était même, en fait, un peu supérieur. De plus, ce phénomène devrait se traduire par un défaut de mâles, à l'éclosion, pour les familles en question. Or, sur l'ensemble des deux années, on constate globablement un excès de poussins femelles, mais les 
nombres de $\sigma^{x} \sigma^{x}$ et de $\left.\not \nmid\right\rceil$ éclos dans le premier groupe de familles (excès de mâles « hérissés ") sont, respectivement, de 289 et 349 , alors que les nombres correspondants pour les familles à rapport de ségrégation «normal "sont de 607 et 709 . On vérifie, par un $\chi^{2}$ de contigence (égal à $0, \mathbf{I} 2 \mathrm{I}$ ) qu'il n'y a pas de différence significative de sex ratio entre ces deux groupes.

\section{TABLEAU 4}

Proportions de crêtes en rose "hérissées" et "lisses " chez les descendants suivant le génotype des parents au locus $R$ (souche $L 22)$

I - Croisement ơ " hérissé " $\times$ 우 " hérissée " (familles comportant une ségrégation)

\begin{tabular}{|c|c|c|c|c|}
\hline $\begin{array}{l}\text { Type de croisement } \\
\text { pour le gène } R\end{array}$ & đ " hérissé " & $\sigma$ "lisse $"$ & q " hérissée " & ㅇ "lisse " \\
\hline Un parent au moins RR. & 91 & 26 & 92 & 41 \\
\hline $\mathrm{R} r \times \mathrm{R} r \ldots \ldots \ldots \ldots$ & 209 & 41 & 196 & 87 \\
\hline $\mathrm{R} r \times r r$ ou $r r \times \mathrm{R} r \ldots$ & 6 & 4 & 12 & 5 \\
\hline
\end{tabular}

II - Croisement $\delta$ " hérissé " $x$ 우 "lisse " ou $\delta$ "lisse " $\times$ ㅇ " hérissée "

(familles comportant une ségrégation)

\begin{tabular}{|c|c|c|c|c|}
\hline $\begin{array}{l}\text { Type de croisement } \\
\text { pour le gène } \mathrm{R}\end{array}$ & $\sigma$ " hérissé " & $\hat{o}$ " lisse " & q "hérissée " & 우 "lisse" \\
\hline Un parent au moins RR. & 207 & 129 & 198 & 223 \\
\hline $\mathrm{R} r \times \mathrm{R} r \ldots \ldots \ldots \ldots$ & 138 & $6 t$ & 129 & 103 \\
\hline $\mathrm{R} r \times r r$ ou $r r \times \mathrm{R} r$ ou & & & & \\
\hline $\mathrm{RR} \times r r \ldots \ldots \ldots \ldots$ & 18 & $t_{k}$ & 27 & 6 \\
\hline
\end{tabular}

Il ne resterait qu'une autre hypothèse possible, celle d'une ségrégation anormale à la méiose ou à la fertilisation. Quoiqu'on ne puisse la rejeter totalement tant que le génotype d'un nombre suffisant de $\sigma^{x} \sigma^{x}$ n'aura pas été vérifié par un progeny test (cf. MÉrat, I964), il serait prudent de ne l'accepter que si nous étions contraints à rejeter la première.

d) Conclusion.

Quant aux observations sur les poussins, toutes nos données confirment, en fin de compte, l'hypothèse de deux allèles avec dominance, le récessif n'ayant une pénétrance complète que chez les femelles.

Secondairement, la population "Jouy ", en ségrégation également pour la crête en rose et la crête simple d'une part, pour le caractère " hérissé " et " lisse " d'autre part, nous a permis de vérifier à nouveau les proportions des deux formes de crêtes 
en rose, suivant le phénotype des parents. Les résultats (réunis pour les années Ig63 et Ig64) sont les suivants, en ne retenant que les familles qui présentaient une ségrégation pour le caractère en question (tab1. 5).

\section{TABI,EAU 5}

Proportions par type de croisement

(souche "Jouy")

\begin{tabular}{|c|c|c|c|c|c|c|}
\hline \multirow{3}{*}{ Type de croisement } & \multicolumn{6}{|c|}{ Proportions chez les descendants } \\
\hline & \multicolumn{2}{|c|}{30} & \multicolumn{2}{|c|}{ 우우 } & \multicolumn{2}{|c|}{ Total } \\
\hline & "hérissés 》 & " lisses " & "hérissées" & " lisses " & " hérissés" & "lisses" \\
\hline 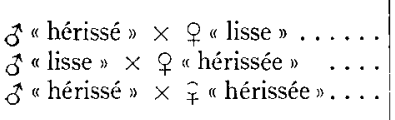 & $\begin{array}{l}49 \\
32 \\
12\end{array}$ & $\begin{array}{r}3 / 4 \\
35 \\
7\end{array}$ & $\begin{array}{l}40 \\
31 \\
16\end{array}$ & $\begin{array}{r}42 \\
43 \\
4\end{array}$ & $\begin{array}{l}89 \\
63 \\
28\end{array}$ & $\begin{array}{l}76 \\
78 \\
11\end{array}$ \\
\hline
\end{tabular}

Ici encore, les observations s'accordent de façon satisfaisante avec notre interprétation monofactorielle.

\section{Indépendance de ce locus et du locus $R$}

Les ségrégations au locus $\mathrm{R}$ (crête en rose/crête simple) existant dans les deux populations étudiées permettent d'exclure 1'hypothèse - que l'on pouvait a priori envisager - d'un allélisme, ou d'un linkage étroit, entre les facteurs " hérissé » et "lisse " et le gène $\mathrm{R}$.

En effet, les deux types de crête en rose s'observent chez des animaux homozygotes RR : nous l'avons vérifié sur la souche $L 22$, et, ultérieurement, sur un troupeau de Wyandottes pures. Ceci obligerait à imaginer deux allèles pour la crête en rose, l'un correspondant au caractère " hérissé ", l'autre au phénotype "lisse ". Mais, dans un croisement, par exemple, entre un hétérogyzote $\mathrm{R} r$ et un récessif $r r$, on ne devrait alors jamais avoir, chez les enfants, qu'un seul type de crête en rose. Or, nos deux populations nous fournissaient de tels croisements, avec une ségrégation parmi les descendants à crête en rose.

Pour ce que ces ségrégations soient compatibles avec un multiallélisme au locus $\mathrm{R}$, il faudrait supposer l'existence simultanée de deux allèles $\mathrm{R}$ et de deux allèles $r$, soit RI, R2, $r 1, r 2$, avec R2 et $r 2$ permettant 1'apparition du phénotype " lisse " chez les crêtes en rose. Nous allons montrer, en détaillant les diverses possibilités concevables de dominance entre ces allèles hypothétiques, que cette hypothèse, d'ailleurs compliquée, ne s'accorde pas, elle non plus, avec les faits.

Supposons que Rr suffise à déterminer le phénotype " hérissé " chez l'hétérozygote $\mathrm{R} r$, quel que soit l'allèle $r$ présent : Les croisements $\mathrm{R} r \times r r$ où le parent à crête en rose est " hétissé " ne devraient jamais donner que des enfants à crête en rose " hérissée ", ce qui n'est pas le cas. 
$\mathrm{Si}$, par contre, RI $r 1$ est le seul génotype Rr permettant la crête " hérissée ", les croisements $\mathrm{R} r \times \mathrm{R} r$ où les deux parents sont " hérissés " ne devraient jamais donner que des enfants " hérissés ". Il devrait en être de même si, enfin, ce phénotype pouvait correspondre, soit au génotype $\mathrm{R} I r 1$, soit à $\mathrm{R} 2 r 1$. Or, il n'en est rien.

Nous proposons donc de désigner les deux allèles étudiés ici, respectivement, par $\mathrm{He}^{+}$(correspondant aux deux premières lettres de " hérissé ») et par $\mathrm{He}^{l}{ }^{1}$ ).

Nous devons admettre que le locus He est distinct de R, et ne lui est pas étroitement lié.

Quant à une estimation précise d'un linkage éventuel, elle est rendue difficile par le fait que l'expression des allèles $\mathrm{He}^{+}$et $\mathrm{He}^{l}$ en présence de la crête simple n'est pas connue. Cependant, un linkage devrait entraîner une hétérogénéité entre familles pour les proportions de crêtes en rose "hérissées " et " lisses ", suivant le sens de la liaison chez les parents doublement hétérozygotes. Une telle hétérogénéité n'existe pas dans nos données, au moins chez les descendants femelles, où la pénétrance du récessif paraît complète. Il faut donc conclure, soit à l'indépendance totale des deux gènes, soit à un linkage trop lâche pour être décelé.

Nous mentionnerons, pour terminer, des proportions de ségrégations observées quand l'un des parents est à crête simple $(r r)$. Nous nous sommes limités au cas où le parent à crête en rose est " lisse ": S'il y a alors ségrégation, on doit conclure que le parent à crête simple est hétérozygote $\mathrm{He}^{+} \mathrm{He}^{l}$. Le résultat est le suivant (tabl. 6).

TABLEAU 6

Ségrégation pour les caractères "hérissées》 et "lisses" quand l'un des parenls est à crête simple

\begin{tabular}{|c|c|c|c|c|}
\hline \multirow{3}{*}{ Croisement } & \multicolumn{4}{|c|}{ Nombre de descendants à crête en rose } \\
\hline & \multicolumn{2}{|c|}{$\theta 0$} & \multicolumn{2}{|c|}{ 우우 } \\
\hline & "hérissés" & "lisses" & " hórissées" & "lisses" \\
\hline $\mathrm{R} r \mathrm{He}^{l} \mathrm{He}^{l} \times r r \mathrm{He}^{+} \mathrm{He}^{l}$ & 63 & 41 & 77 & 39 \\
\hline$r r \mathrm{He}^{+} \mathrm{IIe}^{l} \times \mathrm{R} r \mathrm{He}^{l} \mathrm{He}^{l}$ & $1^{\prime} t$ & 10 & 9 & 1'́t \\
\hline Total . . . . . . . . . & 77 & 51 & 86 & 53 \\
\hline
\end{tabular}

L'excès de femelles "hérissées " issues du croisement $\mathrm{R} r \times r r$ est significatif. Nous n'en avons pas d'explication; dans tous les croisements où la mère est à crête en rose, les proportions sont normales, comme nous l'avons vu précédemment. Une pénétrance incomplète du caractère " lisse " chez les femelles ne semble donc pas une interprétation très satisfaisante. D'autre part, le taux d'éclosion des familles correspondantes n'est pas inférieur à la moyenne du troupeau.

\section{Autres particularités visibles associées au gène $\mathrm{He}$}

Nous n'avons pas, actuellement, de données suffisantes pour établir avec certitude l'existence de différences visibles liées à l'expression des allèles $\mathrm{He}^{+}$et $\mathrm{He}^{l}$, chez les animaux à crête simple.

(') Le symbole $h$ existant déjà pour le gène "plumage soyeux " (HUTT, I949). 
Par contre, des mensurations du squelette crânien faites sur des animaux à crête en rose, de constitution connue pour le gène $\mathrm{He}$, se sont révélées intéressantes.

Sur un échantillon de poules âgées d'un an, ayant été utilisées comme reproductrices au printemps I965, les mesures suivantes ont été prises:

- Largeur maximum du crâne (en arrière et en haut des orbites).

- Largeur minimum du crâne (au-dessus et en avant des orbites).

- Longueur du crâne, de l'extrémité supérieure des fosses nasales à l'extrémité postérieure de l'occipital.

- Hauteur totale maximum du crâne.

En outre, ces animaux avaient été pesés vivants. Leur nombre était de 16 pour les " hérissés " et de ro pour les " lisses ".

I es résultats sont contenus dans le tableau 7 .

TABLEAU 7

Mesures du crâne comparées pour des poules d'un an à crête en rose "hérissée " ou "lisse"

\begin{tabular}{|c|c|c|c|c|}
\hline \multirow[b]{2}{*}{ Mesure } & \multicolumn{2}{|c|}{ Valeur moyenne (en cm.) } & \multirow[b]{2}{*}{$l$} & \multirow[b]{2}{*}{ Probabilité } \\
\hline & $\begin{array}{c}\text { "hérissées" } \\
\left(\mathrm{n}_{1}=16\right)\end{array}$ & $\begin{array}{c}\text { "lisses" } \\
\left(\mathrm{n}_{2}=10\right)\end{array}$ & & \\
\hline Largeur maximum du cràne & 2,95 & 2,83 & 3,076 & $<0,001$ \\
\hline Largeur minimum dı cráne & 1,67 & 1,63 & 0,677 & $>0,5$ \\
\hline I.ongueur du crâne . . . . . & 4,95 & 4,81 & 1,508 & $>0,10$ \\
\hline Hauteur du crâne...... & 3,10 & 2,99 & 2,166 & $<0,05$ \\
\hline
\end{tabular}

Certaines dimensions du crâne diffèrent donc suivant la constitution génétique des poules au locus He. Dans l'ensemble, la tête est un peu plus grande pour celles possédant une crête " hérissée "que pour celles à crête "lisse ". Cependant, dans l'échantillon mesuré, les poids moyens des oiseaux différaient: I 9 Ig g pour les premiers, I $754 \mathrm{~g}$ pour les seconds (différence significative au seuil $5 \mathrm{p}$. Ioo). Pour savoir si l'écart des mensurations crâniennes ne reflétaient pas simplement une différence générale de taille, nous avons fait une analyse de covariance des largeurs maximum du crâne en prenant le poids corporel comme variable indépendante (tabl. 8). Le résultat montre qu'il doit bien exister un effer local associé au gène He. Nos observations peuvent être rapprochées de celles de FiSHER (I938) sur la largeur de la tête comparée chez des animaux à crête simple et à crête en rose : Il est intéressant de noter que deux gènes influant sur la forme de la crête ont également une répercussion sur la morphologie du crâne.

\section{4. Éléments de la "valeur sélective " associée aux allèles $H e^{+}$et $H e^{1}$}

a) Fertilité des mâles.

Sur les trois années I963, I964 et I965 de la population principale étudiée (souche $L$ 22), 4I ơ $^{x}$ à crête "hérissée " et I7 à crête "lisse ", au total, avaient été reproducteurs. Le nombre d'œufs incubés par père s'échelonnait entre 73 et 225 . 
La fertilité moyenne du premier groupe apparaissait moins bonne que celle du second. Cette tendance est reflétée par le tableau 9. Pour chaque année et pour l'ensemble, la moyenne non pondérée des pourcentages par père d'œufs notés "clairs " au mirage est donnée pour les deux catégories, ainsi que celle des pourcentages de poussins éclos rapportés aux œufs mis en incubation.

\section{TABIEAU 8}

Analyse de covariance des largeurs maximales du crâne (variable indépendante $=$ poids corporel)

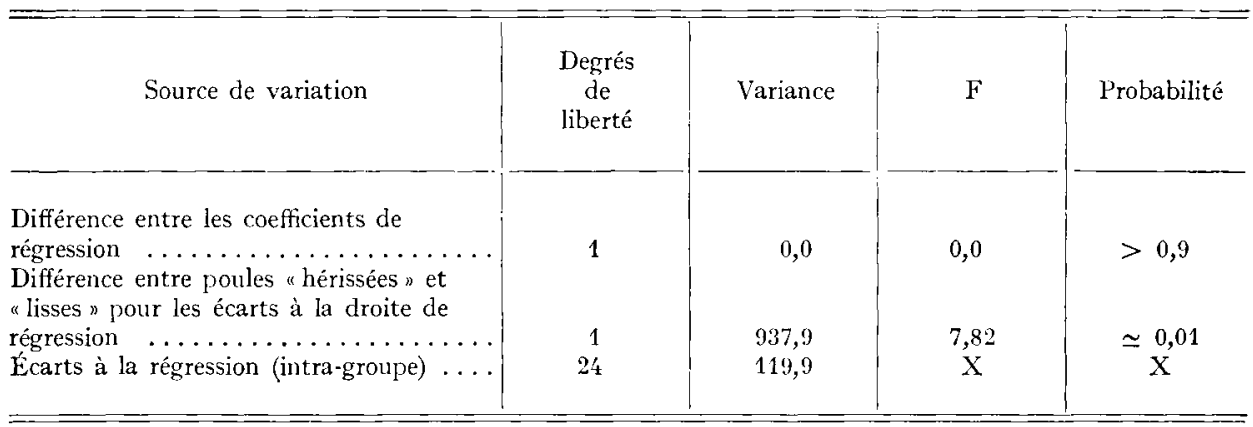

L'examen des nombres d'œufs "clairs " pour chaque coq, outre la différence entre pères " hérissés ) et “ lisses ", suggérait une plus grande variabilité des premiers : six d'entre eux, sur l'ensemble des trois années, donnaient plus de 20 p. roo d'œufs clairs (les proportions s'échelonnant entre 20 et Ioo p. IOo), ce qui n'était le cas pour aucun coq " lisse ".

Il n'apparaissait pas, dans l'ensemble, de différence nette dans le taux de mortalité embryonnaire. Pour une analyse détaillée, nous nous limitons donc au pourcentage d'œufs " clairs », ou plus précisément à sa valeur transformée en angle, pour chaque coq, de façon à rendre la distribution plus proche de la normalité.

Chaque incubation comprenait des ceufs conservés de $\mathrm{x}$ à $2 \mathrm{I}$ jours avant la mise à couver. Ia mortalité embryonnaire était plus grande sur les œufs conservés 3 semaines, mais le taux d'œufs "clairs " était peu affecté, de sorte que nous pouvons ignorer ici ce facteur de variation.

La fertilité moyenne des coqs RR s'est révélée un peu moins bonne que celle des hétérozygotes $\mathrm{R} r$, la différence étant apparemment moins grande que celle observée chez la Wyandotte par Cochez (I95I) et CRAwFord et MERRITT (I963). Comme les premiers étaient peu nombreux et inégalement répartis entre les types " hérissé » et " lisse », nous avons comparé ces deux catégories parmi les seuls coqs $\mathrm{R} r$.

Une analyse de variance à deux facteurs contrôlés (phénotype " hérissé " ou "lisse " des coqs et année) paraissait logique, mais les différences entre années pour le taux de fertilité étant faibles et non significatives, il devenait possible de comparer globalement les deux types de coqs sans tenir compte du dernier facteur. Ceci étant, et comme le suggérait l'examen des pourcentages individuels, il existait une importante différence entre coqs à crête " hérissée " et "lisse " pour la variance de leur taux d'œufs clairs transformé, comme l'indique le tableau ro. 


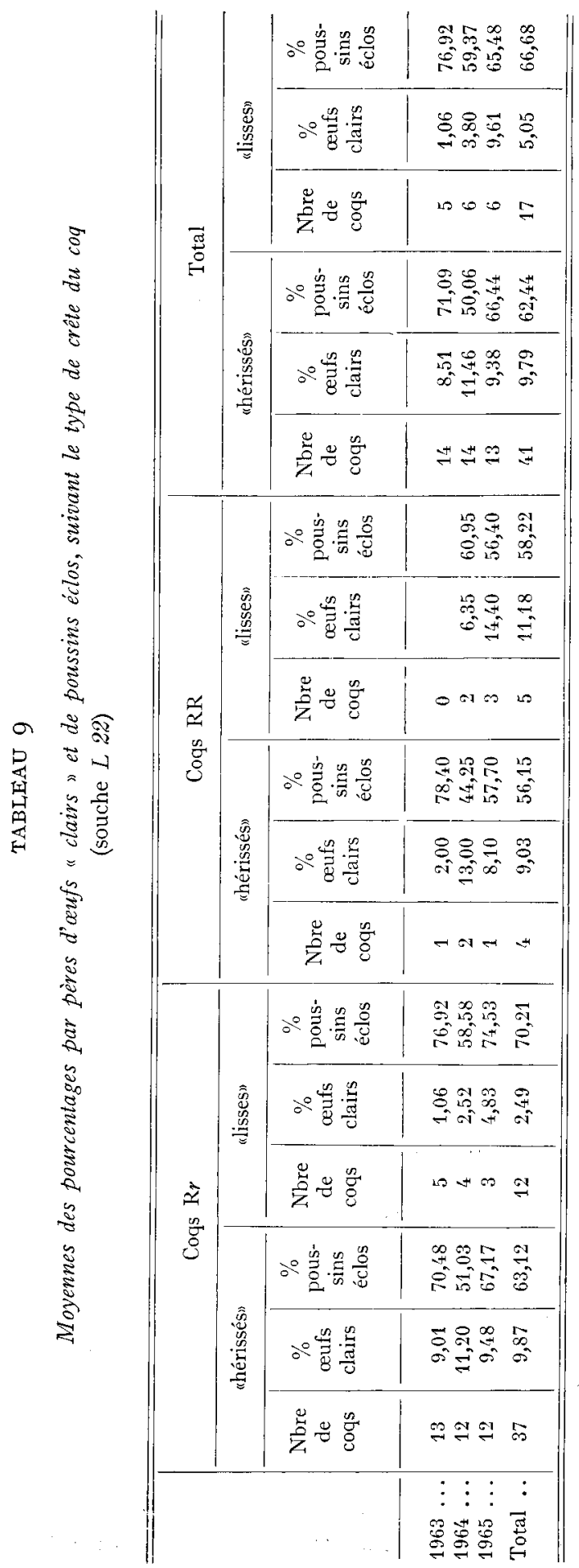


Il n'était donc pas légitime d'utiliser les données telles quelles pour un test $t$, mais nous avons considéré le $t$ calculé de la manière usuelle, comme distribué, du fait de l'hétérogénéité des variances, suivant une loi de STUDENT-FISHER avec un nombre $v$ de degrés de liberté défini par

avec

$$
\frac{\mathrm{I}}{v}=\frac{k^{2}}{v_{1}}+\frac{(\mathrm{I}-k)^{2}}{v_{2}}
$$

$$
\nu_{1}=n_{1}-\mathrm{I}, v_{2}=n_{2}-\mathrm{I} \text { et } k=\frac{s_{1}{ }^{2} / n_{1}}{s_{1}{ }^{2} / n_{1}+s_{2}{ }^{2} / n^{2}},
$$

$n_{1}$ et $n_{2}$ étant les nombres de coqs, $\mathrm{S}_{1}^{2}$ et $\mathrm{S}_{2}^{2}$ les variances des deux groupes (WEI,SCH, cité par Morice et Chartier, I954, p. 207-209).

TABI.EAU IO

Comparaison des variances du pourcentage d'wufs clairs par coq,

\begin{tabular}{|c|c|c|c|c|c|c|}
\hline & \multicolumn{2}{|c|}{ Coqs " hérissés" } & \multicolumn{2}{|c|}{ Coqs "lisses" } & \multirow[b]{2}{*}{$\mathrm{F}$} & \multirow[b]{2}{*}{ Signification } \\
\hline & $\begin{array}{c}\text { Degrés de } \\
\text { liberté }\end{array}$ & Variance & $\begin{array}{c}\text { Degrés de } \\
\text { liberté }\end{array}$ & Variance & & \\
\hline $\begin{array}{c}\text { Coqs RR } \\
\text { et } \mathrm{Rr} \text { groupés }\end{array}$ & 40 & 263,3 & 16 & 70,1 & 3,76 & $P<0,005$ \\
\hline Coqs Rr seulement & 37 & 280,1 & 11 & 26,9 & 10,43 & $P<0,001$ \\
\hline
\end{tabular}
après transformation angulaire arc sin

On obtient ainsi un $\mathrm{t}$ égal à 2,I94 pour un nombre de degrés de liberté voisin de 45 , d'où une probabilité inférieure à 0,05 .

Sur la deuxième population étudiée (souche "Jouy "), l'année rg64 donnait le résultat suivant pour deux coqs "hérissés " comparés à quatre coqs " lisses " de même origine: sur 206 œufs pondus par les poules accouplées aux premiers, 40,3 p. Ioo étaient "clairs ", contre I4,o p. Ioo sur 607 œufs pour les seconds. Ce résultat, quoique portant sur de petits nombres, va dans le même sens que ceux de la souche $L$ 22.

La téalité d'une différence entre coqs à crête en rose " hérissée " et " lisse " pour le pourcentage d'œufs " clairs » qu'ils donnent est donc probable. Cette différence est bien à attribuer aux coq̣s eux-mêmes, car les poules leur étaient accouplées de façon a priori quelconque. Ces œufs " clairs " sont en majorité des zygotes non fécondés, quoiqu'un simple mirage ne puisse garantir une distinction certaine avec des embryons morts très tôt.

La plupart des coqs à phénotype " hérissé " étaient des hétérozygotes $\mathrm{He}^{+} \mathrm{He}^{l}$. Seuls deux d'entre eux se sont révélés être homozygotes $\mathrm{He}^{+} \mathrm{He}^{+}$(en r965), et leurs taux de fertilité étaient parmi les moins bons. Ceci suggère de vérifier s'il existe une différence entre ces deux génotypes.

b) Fertilité et taux d'éclosion des mères.

Nous n'avons pu déceler une différence de fertilité ou de mortalité embryonnaire suivant le génotype de la mère au locus $\mathrm{He}$, vu la nécessité de tenir compte de l'in- 
fluence du père, et le nombre assez réduit de comparaisons " intra-père " pour des mères différentes, que nous avons pu réunir jusqu'ici. Le taux d'éclosion global est moins bon pour les mères homozygotes $\mathrm{He}^{+} \mathrm{He}^{+}$que pour les $\mathrm{He}^{l} \mathrm{He}^{l}$ pour 4 comparaisons de cette sorte sur 6 (taux moyens d'éclosion : 69,9 et 58,o p. Ioo respectivement). Peut-être en est-il de même, à un moindre degré, pour les mères $\mathrm{He}^{+} \mathrm{He}^{l}$ vis-à-vis des $\mathrm{He}^{l} \mathrm{He}^{l}$, mais des données supplémentaires sont nécessaires pour le confirmer.

c) Autres performances.

Nous avons voulu, en outre, comparer, pour les deux phénomènes étudiés, les critères suivants :

Croissance pondérale évaluée par le poids à 8 semaines d'âge.

Nombre d'œufs pondus, de l'entrée en ponte au 3I décembre.

Poids moyen des œufs à l'âge de IO-II mois.

Poids des poules à l'âge de Io-II mois.

TABI,EAU II

Comparaison des performances par la méthode des couples

\begin{tabular}{|c|c|c|c|c|}
\hline \multirow{2}{*}{ Performance } & \multirow{2}{*}{$\begin{array}{l}\text { Nombre de } \\
\text { couples }\end{array}$} & \multicolumn{2}{|c|}{ Valeurs moyennes } & \multirow{2}{*}{$\begin{array}{c}\text { Test de } \\
\text { signification }\end{array}$} \\
\hline & & $\begin{array}{l}\text { Animaux à crête } \\
\text { "hérissée" }\end{array}$ & $\begin{array}{c}\text { Animaux à crête } \\
\text { "lisse " }\end{array}$ & \\
\hline \multicolumn{5}{|l|}{ Poids à 8 semaines des $\widehat{\partial} \widehat{\partial}(\mathrm{g})$} \\
\hline Souche $L 22 \ldots \ldots \ldots \ldots \ldots$ & 161 & 685,7 & 700,8 & N.S, \\
\hline Souche "Jouy" $\ldots \ldots \ldots \ldots \ldots$ & 22 & 864,5 & 856,9 & N.S. \\
\hline 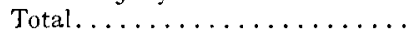 & 183 & 707,2 & 719,6 & N.S. \\
\hline \multicolumn{5}{|l|}{ Poids à 8 semaines des 우우 $(\mathrm{g})$} \\
\hline Souche $L 22 \ldots \ldots \ldots \ldots \ldots$ & 170 & 610,1 & 614,7 & N.S. \\
\hline Souche "Jouy" . . . . . . . . . . & 32 & 770,6 & 716,9 & N.S. \\
\hline 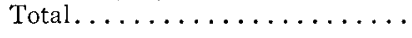 & 202 & 635,6 & 630,9 & N.S. \\
\hline \multicolumn{5}{|l|}{ Nombre d'œufs } \\
\hline Souche $L 22 \ldots \ldots \ldots \ldots \ldots$ & 45 & 37,00 & 38,36 & N.S. \\
\hline Souche "Jouy" . . . . . . . . . . & 14 & 20,29 & $33,6^{\prime} \pm$ & N.S. \\
\hline Total $\ldots \ldots \ldots \ldots \ldots \ldots$ & 59 & 33,37 & 37,24 & N.S. \\
\hline \multicolumn{5}{|l|}{ Poids moyen des cufs (g) } \\
\hline Souche $L 22 \ldots \ldots \ldots \ldots \ldots$ & 29 & 49,10 & 50,62 & N.S. \\
\hline Souche "Jouy" . . . . . . . . . & 15 & 53,22 & 53,77 & N.S. \\
\hline 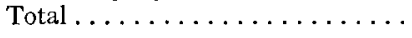 & 44 & 50,50 & 51,69 & N.S. \\
\hline \multicolumn{5}{|l|}{ Poids des poules adultes $(\mathrm{g})$} \\
\hline Souche "Jouy" ........... & 34 & 2098 & 2249 & N.S. \\
\hline
\end{tabular}

Nous avons, à cet effet, constitué des couples d'animaux de même sexe, frères ou sœurs, nés le même jour. Les résultats portent sur les années I963 et r964 de la souche $L 22$ (sauf pour le poids adulte), et l'année I964 de la population 
"Jouy ", ainsi que sur l'année I 965 pour le poids à 8 semaines. Comme les différences entre membres d'un même couple ne semblent pas varier de façon notable suivant l'année, nous nous contentons de donner les valeurs moyennes sur l'ensemble des données, pour chaque caractère (tabl. II).

Aucune des différences n'est significative. On peut cependant noter que la tendance à un poids à 8 semaines légèrement plus grand chez les $\sigma^{x} \sigma^{x}$ " lisses " s'observe dans chacune des années. Peut-être des données ultérieures plus nombreuses pourrontelles confirmer sa réalité.

Pour la mortalité des poulettes, de l'âge de 8 semaines à ro-I I mois, elle était assez faible, dans la souche $L 22$, pour l'une et l'autre des deux catégories comparées

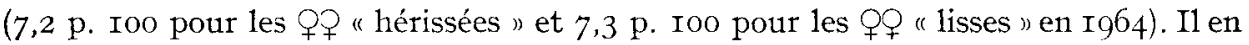
est de même de la mortalité des jeunes.

Enfin, l'observation des embryons morts après I 8 jours d'incubation, en I 964 et $I 965$, pour la souche $L 22$, permettait de comparer le taux de mortalité des embryons des deux phénotypes dans les derniers stades de la vie embryonnaire. Le tableau r2 donne le résultat par type de croisement (les sexes ont été groupés, car il n'apparaissait pas entre eux de différence sensible).

TABLEAU I2

Mortalité après le $18^{\mathbf{e}}$ jour d'incubation comparée pour les embryons à crête "hérissé" ou "lisse" (souche $L 22$, années i 964 et ig65 groupées)

\begin{tabular}{|c|c|c|c|c|}
\hline Type de croisement & Phénotype & Éclos & $\begin{array}{l}\text { Morts après } \\
18 \text { jours }\end{array}$ & $\begin{array}{l}\% \text { de déchet } \\
\text { en éclosoir }\end{array}$ \\
\hline $\begin{array}{c}\mathrm{He}^{+} \mathrm{He}^{l} \times \mathrm{He}^{l} \mathrm{He}^{l} \\
\text { ou } \mathrm{He}^{l} \mathrm{He}^{l} \times \mathrm{He}^{+} \mathrm{He}^{l}\end{array}$ & $\begin{array}{l}\text { "Hérissé " } \\
\text { "Lisse " }\end{array}$ & $\begin{array}{l}540 \\
479\end{array}$ & $\begin{array}{l}70 \\
35\end{array}$ & $\begin{array}{r}11,46 \\
6,81\end{array}$ \\
\hline $\mathrm{He}^{+} \mathrm{He} e^{l} \times \mathrm{He}^{+} \mathrm{He}^{l}$ & $\begin{array}{c}\text { " Hérissé " } \\
\text { " Lisse " }\end{array}$ & $\begin{array}{l}587 \\
205\end{array}$ & $\begin{array}{l}8{ }^{\prime} \\
22\end{array}$ & $\begin{array}{l}12,52 \\
10,73\end{array}$ \\
\hline
\end{tabular}

La différence du taux de mortalité dans le premier cas est significative au seuil I p. roo $\left(\chi^{2}\right.$ de contingence $=7$, I 4 pour I degré de liberté). Pour le deuxième type de croisement, quoique non significative (en partie du fait de l'effectif plus faible des zygotes " lisses ") elle est de même sens, de sorte que, sur l'ensemble des données, on peut conclure à un déchet d'éclosion un peu plus grand pour les embryons à crête " hérissée ".

\section{CONCLUSIONS}

I'intérêt principal du gène mis en évidence est son incidence non négligeable sur le pourcentage d'œufs " clairs " donné par les coqs, qui reflète, au moins approximativement, leur fertilité en accouplement naturel. 
Le déterminisme génétique de ce dernier critère, précisément, est complexe et mal connu. Son héritabilité est faible (cf. par exemple, JuLL., I952). Il est d'autant plus intéressant de connaitre un gène associé à une différence assez importante de fertilité qu'à l'heure actuelle, seuls, à notre connaissance, le locus R (CoCHEz, I95 I ; CRAWFORD et MERRITT, I963) et, peut-être, certains gènes de groupe sanguin (ALLEN, I962; DE SILVA, I965) sont dans ce cas. On peut y ajouter l'effet sans doute direct de la morphologie dans le cas de l'absence de croupion (HuTt, 1949).

Le locus He est-il également associé à un effet sur la fertilité chez des animaux à crête simple? Des recherches sont poursuivies pour le savoir, ainsi que pour essayer d'identifier de façon visible le gène en question dans ce dernier cas; ceci aurait un intérêt certain pour les sélectionneurs de nombreuses souches, auxquels se pose un problème de fertilité.

Il semble curieux que certaines analogies apparaissent entre les effets associés à He et $\mathrm{R}$, qui sont deux gènes de forme de la crête. Comme le second, le premier est lié, d'après ce qui précède, à des différences de fertilité des mâles, et sans doute de la morphologie du squelette crânien. Il resterait à déterminer si cette analogie n'est qu'accidentelle, ou, sinon, quelle est sa raison d'être du point de vue physiologique.

Nous avons pu constater que les deux allèles $\mathrm{He}^{+}$et $\mathrm{He}^{l}$ sont présents, outre les souches $L 22$ et "Jouy ", dans une lignée de Wyandottes, et dans une souche à crête simple, issue au départ d'un croisement entre Gâtinaise, Rhode-Island et Wyandotte. Un sondage préliminaire nous a encore révélé leur existence chez des Faverolles, et dans un croisement (Heisdorf-Nelson) .

Le troupeau $L 22$ de 1963 était le premier où le locus He était étudié ; par conséquent, aucun choix conscient n'avait pu y être opéré sur les génotypes à ce locus : la fréquence de l'allèle $\mathrm{He}^{l}$, déduite de la proportion des deux phénotypes observés, y est égale à 0,64 .

Il est trop tôt pour savoir si le polymorphisme trouvé dans ces diverses populations est fortuit. ou s'il existe des mécanismes tendant à le maintenir. L'examen comparé des performances suggère, jusqu'ici, un avantage sélectif pour le caractère " lisse ", mais la question de savoir si l'hétérozygote ne possède pas certains avantages ne peut encore recevoir une réponse.

Reçu pour publication en septembre 1965 .

\section{SUMMARY}

A NEW GENE, MODIFYING THE FORM OF ROSE COMBS, AND ITS POSSIBLE ASSOCIATION WITH COCK FERTILITY

A new comb-type gene is identified in a pedigree population whose origin is a cross between Leghorns, Rhode-Island Reds and Wyandottes. The effect of this gene is apparent in rose-comb birds. It has two alleles, which we called $\mathrm{He}^{+}$(" hérissé" = rugged) and $\mathrm{He}^{l}$ ("lisse " = smooth). The first one is dominant. In day-old chicks, it gives a granular appearance to rose-combs. On the contrary, these rose-combs have a smooth surface in birds homozygous for the second allele (fig. I). In adults, the $\mathrm{He}^{+}$gene causes the comb to be more bulky, with more numerous and higher spikes than $\mathrm{He}^{l}$ (fig. 2). The difference between the two phenotypes is more evident in day-old chicks than in adult birds. 
The pénétrance of the recessive allele at one day seems to be complete among females, but not among males. This is shown by the observed proportions in all the possible mating types (table 1 )

For female progeny, these proportions are in good agreement with the expectation, and there is no heterogeneity between families (table 2). This does not hold for males, suggesting the existence of genes modifying the pénétrance of the $\mathrm{He}^{l}$ gene in that sex (table 3 ).

These data, together with matings made in another population with one parent being single combed, show that the locus studied is different from the rose/single comb locus and is probably not linked with it. ble 7 ).

Skeletal measurements suggest that this gene could have an effect on skull morphology (ta-

An appreciable difference in male fertility was found to be associated with that gene, " smoothcombed " $\left(\mathrm{He}^{l} \mathrm{He}^{l}\right)$ cocks having, on the average, a higher and less variable fertility percentage than $\mathrm{He}^{+} \mathrm{He}^{+}$or $\mathrm{He}^{+} \mathrm{IHe}^{l}$ ones (tables 9 and $\mathrm{ro}$ ). Research is going on to know whether the same difference of fertility exists among single-comb birds.

We have not found yet any significant difference between birds of the two phenotypes for the following traits : 8-week weight of cockerels and pullets, number of eggs laid from the onset of laying up to 3 Ith december (for spring-hatched pullets), mean egg weight, hatch percentage of dams, chick and adult mortality (table II). On the other hand, "smooth-combed" embryos have a lower mortality rate after the $\mathrm{i} 8$ th day of incubation (table I2).

\section{RÉFÉRENCES BIBLIOGRAPHIQUES}

Alder H. E., 1946. The inheritance of comb variation in the domestic fowl. University of Nebraska, Lincoln, Nebraska.

AlLEN C. P., 1962. The effect of parental B locus genotypes on multiple cross performance in chickens. Ann. N. Y. Acad. Sci., 97, 184-193.

Cochez L. P., i95ı. Un facteur d'infertilité, équilibré par la sélection chez la Wyandotte blanche. $C . R$. $X I \mathrm{e}$ Congrès mondial d'Auiculture, Paris, $1,8 \mathrm{I}-88$.

Crawford R. D., MerritT E. S., I953. The relationship between rose comb and reproduction in the domestic fowl. Canad. J. Genel. Cytol., 5, 89-95.

De Silva P. L. G., r 965. Heterozygosity at red cell antigen locus L and fertility. Genetics, 51, 41-48.

Fisher R. A., 1938. Dominance in poultry : Feathered feet, rose comb, internal pigment and pile. Proc. Roy. Soc., B, 125, $25-48$.

Hutr F. B., 1949. Genetics of the fowl. MeGraw Hill Book Co, New York.

Jule M. A., I952. Poultry breeding. J. Wiley and Sons, Ltd.

Mérat P., I964. Ségrégations anormales pour les allèles "crête simple " et "crête en rose " chez la poule. IV. Discussion d'ensemble relative aux trois typas de croisement. Ann. Biol. anim. Bioch. Biophys., 3, I33-I4I.

Morice E., Chartier F., I954. Méthode slatistique. Imprimerie nationale, Paris. 